\title{
VET-Artdevivre Partnership Programme: Identification of market and training needs on wine, health and social aspects and innovative tools and common approaches
}

\author{
Stylianos Filopoulos ${ }^{1}$, Nadia Frittella ${ }^{1}$, Ursula Fradera ${ }^{2}$, Claudia Stein-Hammer ${ }^{2}$, Panagiotis Tataridis ${ }^{3}$, Stylianos Logothetis ${ }^{3}$, \\ Sofoklis Petropoulos ${ }^{3}$, Andreas Matthidis ${ }^{4}$, and Vasiliki Koutsovoulou ${ }^{4}$ \\ ${ }^{1}$ Wine in Moderation-Art de Vivre (WIM) Aisbl, Avenues des Arts 43, 1040 Bruxelles, Belgium \\ ${ }^{2}$ Deutsche Weinakademie (DWA), Gutenbergplatz 3-5, 55116 Mainz, Germany \\ ${ }^{3}$ Panhellenic Union of Registered Oenologists (PANEPO), Verantzerou 15, 10677 Athens, Greece \\ ${ }^{4}$ Union de la Sommellerie Grecque (P.EN.O), Achaion 27, 17564, P. Faliro, Greece
}

\begin{abstract}
Introduction: The VET-ArtdeVivre Partnership Programme was a two-year Partnership launched in August 2012 and funded by the EC's Leonardo da Vinci (LdV). The Partnership brought together nine different actors from seven countries of both the wider wine value chain and the Vocational Education \& Training (VET) field. The work focused on five different topics. The main objective was to identify market $\&$ training needs and to develop common approaches on wine, health $\&$ social aspects. Methods: A Market and Training Needs Analysis (MTNA) Report, consisting of the respective national versions, was drafted by the Partners and reviewed by international experts. The MTNA Report provided an in depth analysis of the respective wine markets and VET, an assessment of selected key job profiles and a mapping of relevant VET offers. Results: Based on the MTNA Report, the Partnership has developed a VET framework proposal with three different training modules according to the professional needs of Sommeliers (EQF 3-5 level), Oenologists (EQF 6 level), and Physicians (EQF 6-8 level). Each module lists the expected learning hours and outcomes, the delivery and assessment methods, reading list, the trainers' minimum formal qualifications and experience required. These proposed comprehensive training modules are linked to a database of existing training references. Considering the MTNA results and having established a concrete proposal, the Partnership has initiated the necessary dialogue to introduce and recognise these skills both at formal \& informal level and national \& EU level in accordance with EQF \& ECVET. Conclusions: Taking into account the increasing interest on the topic and the identified gaps, professional training on wine, health and social aspects and responsible business practices should be considered by the respective stakeholders involved in defining job profiles and training programmes for professionals of the wine value chain.
\end{abstract}

\section{Introduction}

\subsection{Background Leonardo da Vinci Programme}

The VET-ArtdeVivre Partnership Programme was a twoyear Partnership launched in August 2012. The Partnership was funded by the European Commissions' (EC) Leonardo da Vinci (LdV) [1].

The LdV was a sub-programme of the Life Long Learning Programme (LLP) that has expired and was replaced in 2014 by the Erasmus+. LDV funded practical projects in the field of vocational education and training. Initiatives ranged from those providing work-related training to individuals, to larger cooperation efforts. The LdV Partnerships were small-scale cooperation projects between organisations working in the field of Vocational Education and Training (VET), on a theme(s) of common interest. The projects run for a fixed period of two years.

\subsection{Background Wine culture and political environment}

Wine is an integral part to European life and culture. The sector permeates many levels of EU life, contributing significantly to society in socio-economic, environmental \& societal terms: where vines grace the landscape, the wine sector provides employment to millions, helping to sustain the fabric of rural societies and maintaining a way of life that is central to the very notion of European identity. Wine production is an essential economic and labour intensive activity for many regional economies and plays an important role in rural areas of many EU Member States and their regions. The socioeconomic dimension of viti-viniculture extends beyond the agricultural activity in the vineyards or the wineries since other economic activities are directly linked to wine production providing jobs to hundreds of thousands of people across the EU (i.e. trade and marketing of wine, production of oak casks, bottles, labels, capsules, corks, etc., development of wine tourism, production of spirits, distillation of wine and wine by-products - and indirectly linked activities like Hotel, Restaurant, Catering (HORECA), retail, etc.). The wine sector's importance to the EU economy should not be counted merely in monetary terms since it contributes considerably to the EU environment and to the cultural and culinary heritage. Wine products are enjoyed by millions in Europe and around the world. The key place of moderate wine consumption paired with meals has been recognised as a key ingredient of the "Mediterranean Diet" [2] 
and the "Gastronomic meal of the French" [3] both have been incorporated in the UNESCO representative list of Intangible Cultural Heritage of Humanity.

Even though for the majority of consumers, alcoholic beverages, and more specifically wine products, represent a pleasurable beverage to be enjoyed in moderation, there is a minority of the European population that abuses alcoholic beverages in ways that are damaging to themselves and that can harm others around them [4]. Since there is no single European way to consume wine, drinking patterns may depend greatly upon local traditions, religion, education, gender, age group and socioeconomic factors. It is widely recognised that drinking patterns of the various alcoholic beverages specifically vary among different parts of the EU, and among certain social, age \& socioeconomic groups. Enormous cross-cultural variations also show how Europeans behave when they drink [5]. Also, as stated in the "EU strategy to support Member States in reducing alcohol-related harm" [6], harmful and hazardous alcohol consumption has a major impact on public health and also generates costs related to health care, health insurance, law enforcement and public order and workplaces, and thus, has a negative impact on economic development and on society as a whole. Harmful and hazardous alcohol consumption is a key health determinant and one of the main causes of premature death and avoidable disease. It is a net cause of $7.4 \%$ of all ill-health and early death in the EU, and has a negative impact on labour and productivity. It is not alcohol per se but the harmful alcohol consumption that creates a problem. On the other hand, moderate consumption of alcoholic beverages appears to offer protection against coronary heart disease among elderly people (45 \& above) depending on gender and individual differences. Consistently, findings of scientific studies show that low to moderate consumption of wine is associated with a lower overall health risk and that abusive consumption highly increases the risks [7-11].

To reduce alcohol related harm, the EU adopted a strategy to support Member States in reducing alcohol-related harm [6] in 2006, and one of the five priorities of this strategy is to inform, educate and raise awareness on harmful and hazardous consumption and to increase the knowledge among the society about responsible drinking patterns.

At the beginning of the Partnership project, the following factors needed to be considered:

- The Commission's "Communication for an EU strategy to support Member States in reducing alcohol related harm" [6] and the commitment of the authorities, industry and other stakeholders to tackle harm from the abusive consumption of alcoholic beverage and encourage moderate and responsible drinking patterns.

- The concrete and measurable commitment of the EU wine sector to the European Alcohol and Health Forum [12] with the Wine in Moderation - Art de Vivre (WIM) Programme building on the wine culture and backed by science, education and self-regulation to reinforce appropriate drinking patterns as social and cultural norm.

- The growing interest and efforts to provide life-skills to promote healthier lifestyles and the need to increase awareness of consumers on responsible consumption patterns and the risks associated with excessive drinking, will require skilled professionals to properly address the issue to the society and consumers.

- The hundred thousands of professionals in the working at the different stages of the wine economic value chain (e.g. wine production, sales, PR \& marketing, wine writers, wholesalers, retailers, importers/ exporters (wine) tourist operators, sommeliers, barmen, waiters, restaurant owners, chefs, caterers, etc.) who can inform consumers on appropriate drinking patterns.

- The large number of VET establishments across the EU that provide wine appreciation courses to professionals or to the general public offer a unique opportunity to provide training modules on wine, health and social aspects.

- The validation of non-formal and informal learning is still hard to realise in a number of countries [13], making it difficult to certify or recognize at national and EU level, the skills acquired by informal or non-formal training programmes provided by companies to their employees.

- The recommendation of the EU Parliament on the establishment of a European Credit System for Vocational Education and Training (ECVET) [14] intended to facilitate the transfer, recognition and accumulation of assessed learning outcomes of individuals who are aiming to achieve a qualification.

Taking into account all of the factors above, the objective of this Partnership was to identify market and training (VET) approaches in the Partners' countries and to develop common education approaches on wine, health and social aspects.

\section{Methods}

The VET-ArtdeVivre Partnership brought together nine different actors of both the wider wine value chain and the vocational education, with experience and interest in the wine, health and social aspects to:

1) Identify and understand training needs and good responsible practices (responsible selling, serving, and communication) to encourage moderate and responsible drinking patterns and raise awareness on the risks associated with harmful drinking.

2) To propose common approaches compatible with the EU standards on Vocational Education and Training and work towards introducing and recognizing these findings in the European and National Life Long Learning accreditation systems.

3) Establish new working links and improve the quality of cooperation and professional education offer.

The nine Partners from seven different EU Member States were: Wine in Moderation-Art de Vivre (WIM) Aisbl (programme coordinator, Belgium); Deutsche Weinakademie (DWA) (Germany); University of SplitSchool of Medicine, Croatian Chamber of Economy (CCE) (Croatia); Federación Española del Vino (FEV) (Spain); the Unione Italiana Vini (UIV) (Italy); Panhellenic Union of Registered Oenologists (PANEPO), Union de 
la Sommellerie Grecque (P.EN.O) (Greece) and Vin \& Société (V\&S) (France).

With the aim of answering the following questions:

- What are the wine, health and social aspects that should be addressed through vocational education and training?

- What knowledge, skills, competences are needed to solve the problems? Are they adequately developed?

- What are the tools/approaches for developing the necessary skills and how could they be best addressed?

For this purpose, working meetings (mobilities) took place in the Partners' countries. Following the project's obligation, and under the coordination of WIM aisbl, the VETArtdeVivre Partnership defined six working packages i) Partnership management and coordination, ii) Market and Training needs analysis, iii) Database, iv) EU VET framework, v) Validation of Project Results, vi) Dissemination). The working tasks were allocated and a working meeting schedule for the two-year period of the Partnership was set to achieve the objectives.

An in-depth analysis of the national and European market in the form of Market Training Needs Analysis (MTNA) Report was performed considering socioeconomic, alcohol and health issues as well as VET, wine business policies, structures and approaches, alcohol/wine consumption patterns, main problems related to abusive consumption of alcoholic beverages, scientific evidence, identification of relevant key professions to address respective needs, respective training offers and to identify training needs in the area of wine, health and social aspects.

The national job profile descriptions for the identified key professional groups were assessed, including references and gaps in terms of necessary knowledge, skills and competences, in compliance with the European Qualification Framework (EQF) \& European Credit System for VET (ECVET).

Furthermore, both informal and formal VET innovative approaches, references and tools were mapped and a database of good practices was created to support corresponding Training Needs Analysis (TNA) \& ECVET recommendations.

Innovative structured and tangible recommendations and tools based on EQF and ECVET were drafted and proposed to the national and EU VET community and the professionals of the wine sector.

The achieved results were disseminated through targeted events (e.g. working meetings, conferences) and communications to ensure the impact of the results towards the identified target groups and a larger audience.

Through the structured approach of the MTNA Report, the Partners have used the meetings as a way to share their expertise, knowledge and experience to understand in a multi-disciplinary and multi-country approach the market and training needs in their countries and in the EU. Alcohol, Health and Society is indeed a complex topic with huge cultural diversity, therefore a large number of meetings focused on understanding the market issues, both at national and EU level. In parallel, the members carried out a thorough bibliographic and internet research to identify references, such as EU, International, national publications, research and surveys to understand the respective policies, strategies and approaches. This research was also used to identify key stakeholders (Wine value chain, VET, authorities, etc.), and to feed the Partnership's database with innovative VET approaches and tools. Some of the identified key stakeholders (national, EU, International) participated in a number of meetings and events to inform them about the Partnership, to exchange ideas and approaches and to raise the significance of the VET on wine, health and responsible drinking.

The monitoring and the progress of the project was achieved by mapping the outcome of the meetings (mobilities) as well as evaluating the project deliverables by external experts.

\section{Results}

The Partnership project achieved the following results:

A web-space http://artdevivre-edu.eu/, that acts as the focal point of the VET-Artdevivre Partnership with relevant information, news and the results of the Partnership was created.

Based on the Market Training Needs Analysis (MTNA) Report that corresponds to a market analysis on wine, alcohol, health \& social aspects, a Training Needs Analysis for key professions of the wine value chain was created. The MTNA Report [15] is an in-depth and current analysis of the national and EU situation taking into account the diversity of approaches, tools, structures and practices as well as the respective policies and regulatory framework. It maps key professional groups which could play a relevant and critical role in tackling alcohol related problems and in identifying references and gaps in terms of necessary knowledge, skills and competences. It further provides a mapping of both informal and formal VET innovative approaches and offers and presents the various actors in the wine sector concerned by VET activities (provider or beneficiary). The MTNA Report and the respective national MTNA reports are one of the most important deliverables of the Partnership and set the basis for the other Partnership deliverables, but also serve as a valuable and comprehensive source of information for professional actors in the whole wine business and VET area.

After the conclusion of the MTNA report, the Partnership proceeded in identifying the necessary skills, knowledge and competences needed for the wine-related professionals to tackle alcohol-related harm \& promote moderation \& responsibility. The Partnership compiled a list of professions in the wine value chain that could be considered as more relevant for the purpose of the project and to the overall market needs.

Based on the expertise, knowledge, experience and interest of the Partners, it was decided to focus only on one job profile per key group to be analysed further. These job profiles were also chosen because they covered different European Qualification Framework (EQF) levels. These professions were: Sommelier (post-secondary education, 4-5 EQF level), Oenologist (tertiary education, 6 EQF level) and Physician (tertiary education, 6-8 EQF level). The Partnership developed a VET framework proposal [16] with three different training module proposals for these three professions. Each training module describes the expected hours of total learning, the learning outcomes, 
the delivery and assessment methods, the types of reading list, the minimum formal qualifications and experience required by the trainer to deliver the module. A modular approach was chosen by the Partnership since it facilitates the incorporation of such training not only in different EQF levels, but also into a variety of ongoing training programmes. The proposed European VET framework, with the concrete training module proposals for the three respective key professions is available on the Partnership's website and easily accessible.

The online database of corresponding VET references during the research conducted for the MTNA report provides a wealth of information and knowledge. The partnership created an open database to provide references to both, the MTNA report and the VET framework proposal. The database on the Partnership's website lists training programmes, learning outcomes and guidelines, certification schemes, etc. to be used as a reference when designing or providing relevant VET approaches and offers. The database is open to encourage stakeholders to add their own training programme and allow its sustainability and growth.

Having established a concrete proposal, the Partnership has initiated the necessary dialogue to introduce and acknowledge these skills both at formal and informal level and national and EU VET, in line with the EQF and ECVET. A number of events, meetings, conferences aimed at experts and larger audiences have taken place to exchange ideas and disseminate the results. These events were supported with a number of communication activities such as press releases, interviews, on-line posting, etc.

The draft results of the programme (e.g. draft national MTNA reports, VET frameworks, training offer) were presented to external key experts and their feedback was used to assure and validate the results. Key experts/representatives responded to a structured questionnaire about the deliverables of the project and their overall appreciation of the Partnership. In total, thirty experts from nine countries answered the questionnaire (seven partner countries, plus Canada and Australia). Their expertise varied and they worked in different sectors related to the project. Their responses were analysed and integrated in the Partnership's overall results.

The feedback was overall very positive and encouraging for the Partners' efforts. Among the most interesting comments was the need to also include/develop the retail sector in the Partnership's field of interest.

$76 \%$ of the experts considered the analysis of the country overview representative of the country and the mapping of the national relevant training offer/providers complete. $80 \%$ rated their satisfaction about the report with more than 4 on a scale from 1 to 5 , with 1 being the least $\& 5$ the most satisfied.

All the respondents considered that the training modules developed are appropriate and adequate, with the only exception of the physician's module where an addendums was requested. $86 \%$ of the respondents considered that the knowledge, skills \& competences described in the learning outcomes of the training module should be introduced as prerequisite in the job profiles of the relevant professions. $96 \%$ said that they would be interested in using the results of the Partnership in their daily work.

\section{Conclusions and discussions}

The Partnership's analysis showed that there is a gap in the VET offers, especially in the formal ones, on wine, health and responsible drinking, serving, selling in the wine related vocations in the seven Partner countries. Wine professionals, especially those in direct contact with consumers, should be the main target group when considering VET, as they can directly apply their competences and act as multipliers of knowledge and catalysts of behavioural change.

Raising the knowledge, skills and competences of professionals working in the wine value chain on social responsible business practices (responsible serving, selling, and communication) and healthy lifestyles, with innovative training approaches and tools, should be key ingredient for the social responsibility and sustainability of the whole wine business.

All Partners have acknowledged the overall increase of knowledge on VET issues and approaches as well as the ECVET/EQF and their significance in their activities. They have also increased their understanding about the other Partners' national wine markets, about the job profiles of key wine value chain professions and about the alcohol related problems and alcohol policies. The Partnership was complimented as a good forum for exchange of ideas, best practices and approaches as well as a good network opportunity for future common projects and approaches. The Partnership and its tools were very valuable in general and specifically for their respective associations and they have recognised the opportunity to use the outcomes in their ongoing and future activities, ensuring their sustainability and further development.

The National LdV Offices responsible for the monitoring, evaluation and funding of the LDV Partnership Programme have awarded the VET-ArtdeVivre Partnership with very good to excellent scores across all categories, recognizing it as an example of good practice.

All efforts accomplished by this Partnership should be considered by International Institutes and Organisations (EU, OIV, etc.) as well as the national VET authorities when defining VET policies, job profiles and training programmes of related professions (e.g. oenologists and sommeliers). The recent OIV's Strategic Plan 2015-2019 [17], which works towards the definition of the oenologist and sommelier profession and their respective training curriculum provides a key opportunity to introduce respective training modules on wine, health and social aspects and successfully address the above mentioned challenges.

In addition, the results of this partnership should be exploited and implemented through future collaborative projects open to more actors of EU VET community.

\section{References}

[1] European Parliament, European Council, Decision No 1720/2006/EC of the European Parliament and of the Council of 15 November 2006 establishing an action programme in the field of lifelong learning,(2006) http://eurlex.europa.eu/legal-content/EN/ $\mathrm{TXT} /$ ?uri=celex $: 32006 \mathrm{D} 1720$ 
[2] UNESCO, Representative list of Intangible Cultural Heritage of Humanity, "Mediterranean Diet", (2013), http: //www. unesco.org/culture/ich/ $\mathrm{RL} / 00884$

[3] UNESCO, Representative list of Intangible Cultural Heritage of Humanity "Gastronomic meal of the French", (2010) http://www. unesco.org/ culture/ich/index . php?RL=00437

[4] TNS Opinion \& Social, European Commission, Special Eurobarometer 331, EU citizens' attitudes towards alcohol, Wave 72.3 (2010), http:// ec . europa.eu/health/alcohol/docs / ebs_331_en.pdf

[5] H. Leifman, E. Österberg, M. Ramstedt, European Comparative Alcohol Study - ECAS-final report, (2002)

[6] Commission of The European Communities, Communication from the Commission to the Council, the European Parliament, the European Economic and Social Committee and the Committee of the Regions, An EU Strategy to Support Member States In Reducing Alcohol Related Harm (Sec(2006) 1358\} \{Sec(2006) 1360\}\{Sec(2006) 1411), http:// eur-lex.europa .eu/legal-content / EN/TXT/?uri=CELEX : 52006DC0625

[7] M. Roerecke, J. Rehm, Alcohol consumption, drinking patterns, and ischemic heart disease: a narrative review of meta-analyses and a systematic review and meta-analysis of the impact of heavy drinking occasions on risk for moderate drinkers, (2014), BMC Med, 12,1: 182

[8] A. Giacosa, R. Barale, I. Bavaresco, M.A. Faliva; V. Gerbi; C. La Vecchia; E. Negri, A. Opizz, S. Perna, M. Pezzotti, M. Rondanelli, Mediterranean way of drinking and longevity, (2014), Crit Rev.Food Sci.Nutr., 10.1080/10408398.2012.747484 [doi]

[9] S.E Brien, P.E. Ronksley, B.J. Turner, K.J Mukamal, W.A. Ghali, Effect of alcohol consumption on biological markers associated with risk of coronary heart disease: systematic review and meta-analysis of interventional studies, (2011), 342: d636

[10] P.E. Ronksley, S.E Brien; B.J. Turner, K.J. Mukamal, W.A. Ghali, Association of alcohol consumption with selected cardiovascular disease outcomes: a systematic review and meta-analysis, (2011), BMJ, 342: $d 671$

[11] M. Roerecke, J. Rehm, Chronic heavy drinking and ischaemic heart disease: a systematic review and meta-analysis, (2014), Open Heart, 10.1136/ openhrt-2014-000135 [doi];openhrt-2014-000135

[12] European Alcohol and Health Forum, Charter establishing the European Alcohol and Health Forum,(2007), http: / / ec.europa.eu/health/ph determinants/life style/alcohol/ documents/Alcohol_charter2007.pdf

[13] J. Hawley, M. Souto Otero, C. Duchemin, 2010 update of the European Inventory on Validation of Non-formal and Informal Learning - Final Report' (2010)

[14] European Parliament, European Council, Recommendation of the European Parliament and of the Council of 18 June 2009 on the Establishment of a European Credit System for Vocational Education and Training (ECVET) (2009/C 155/02), http://eur-lex. europa .eu/legal-content/EN/ALL/?uri $=$ CELEX $: 32009 H 0708 \% 2802 \% 29$

[15] VET-ArtdeVivre Partnership, Market Training Needs Analysis Report, (2014), http: / / artdevivre-edu.eu/index.php/mk-resultsapproaches/mk-mtna-report . html

[16] VET-ArtdeVivre Partnership, VET-ArtdeVivre Partnership VET framework proposal, (2014), http:// artdevivre-edu . eu/index . php/mkresults-approaches/mk-proposed-vetframework. html

[17] International Organisation of Wine and Vine, OIV's Strategic Plan 2015-2019, (2014), http: / / www . oiv. int/oiv/info/enplanstrategique 